
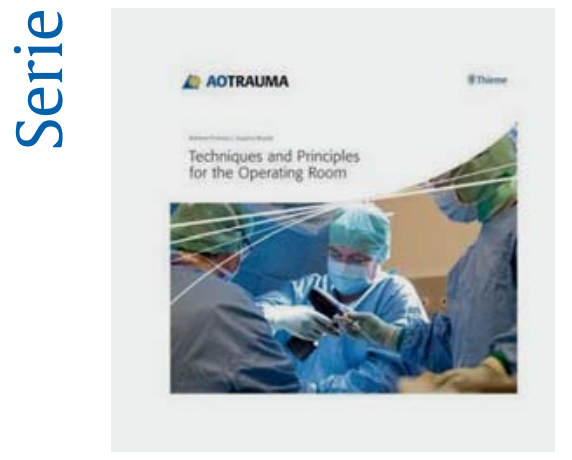

\section{Einleitung}

Der Erfolg bei der operativen Frakturbehandlung ist direkt abhängig von der Qualität der präoperativen Planung des ganzen Teams. Üblicherweise besteht diese Planung in einer Zeichnung des Chirurgen mit detaillierten Abläufen des Eingriffs. Dies erfordert jedoch ein gut integriertes Team innerhalb des Operationsaals und des Pflegepersonals. In gewissen Ländern gibt es derzeit verbindliche Bestimmungen zur Erhöhung der Patientensicherheit, die einen solchen Operationsplan beinhalten. Dieses Kapitel befasst sich mit den Rollen und Aufgaben der verschiedenen Teilnehmer des OP-Teams und den notwendigen Schritten zum Optimieren des chirurgischen Eingriffs.

\section{Warum planen?}

Folgende Vorteile bringt eine sorgfältige präoperative Planung:

- Harmonischer Ablauf des Eingriffs

- Verkürzung der Operationszeit

- Voraussehen und Vermeiden von technischen Problemen

- Erarbeiten von chirurgischen Varianten auf Papier bereits vor dem Eingriff

- Verwirrung und Frustration im OP vermindern

- Sicherstellen der Verfügbarkeit der erforderlichen Implantate und Instrumente

- Vermeiden von unnötigem Öffnen und Resterilisieren von Sets

* Das hier vorliegende Kapitel erschien in englischer Sprache: Techniques and Principles for the Operating Room. Porteous M, Bäuerle S. (C) 2010 by AO Foundation, Switzerland, Clavadelerstr. 8, CH-7270 Davos. Platz Distribution by Georg Thieme Verlag, Rüdigerstr. 14, D70469 Stuttgart and Thieme New York, 333 Seventh Avenue, New York, NY 10001, USA.

** Übersetzung: Prof. Dr. med. Thomas Rüedi, Maienfeld, Schweiz

OP-JOURNAL 2011; 27: 284-287

(C) Georg Thieme Verlag KG Stuttgart · New York DOI http://dx.doi.org/10.1055/s-0031-1280208

\title{
Präoperative Planung für das OP-Personal - als Team*
}

\author{
Steven Schelkun**
}

- Ermöglicht, dass das chirurgische Team den Eingriff im Voraus überdenkt und bespricht

- Ermöglicht, dass zusätzliche Ausrüstung (z.B. Bildwandler oder Cell Saver) bereitstehen samt Bedienung

Ein Team, das tatsächlich regelmäßig plant, kann die Aussage bestätigen, dass „failing to plan means planning to fail“.

\section{Schritte zur Planung}

Die präoperative Planung im Team kann mit dem Erstellen eines Flugplans in der Aviatik verglichen werden. Für jeden kommerziellen Flug erstellt der Flugkapitän einen Flugplan, der in mancher Hinsicht einem chirurgischen Operationsplan entspricht. So wie der Flugplan den nationalen Flugbehörden vorgelegt werden muss, sollte der Chirurg seinen Plan dem ganzen OP-Team, einschließlich Anästhesist, OP-Anästhesielagerungspflege und dem Röntgenassistenten, kommunizieren. Ähnlich wie der Pilot seine Instrumente und Apparate überprüft, muss der Instrumentierende sicherstellen, dass die notwendigen Implantate und Instrumente gemäß Plan und vollständig zur Verfügung stehen. Schließlich wird der Pilot unmittelbar vor dem Start einen nochmaligen „Check“ vornehmen, um das Funktionieren aller Instrumente zu bestätigen. Dementsprechend sollte sich auch der Chirurg vor dem „Schnitt“ mündlich vergegenwärtigen, dass der richtige Eingriff am richtigen Patienten und auf der richtigen, entsprechend markierten Seite vorgenommen wird und dass die schriftliche Einwilligung des Patienten vorliegt.

Arbeiten im Team bedeutet, dass jedes Teammitglied mitdenkt und mithilft und dass auch jederzeit Fragen bezüglich des geplanten Eingriffs erwünscht und gestattet sind. Diese Art von Kommunikation soll die Mitarbeiter einbeziehen, motivieren und steigert deren Interesse am Fall. Während der Chirurg für den
Eingriff als Ganzes verantwortlich ist, trägt jedes Teammitglied wesentlich zum Erfolg bei. Fehler entstehen meist durch mehrere Umstände und wenn die einzelnen Mitglieder des Teams ihre Bedenken nicht zu äußern wagen.

Der chirurgische Plan sollte 4 Stufen beinhalten:

\begin{tabular}{|c|c|}
\hline $\begin{array}{l}\text { Stufe der präope- } \\
\text { rativen Planung }\end{array}$ & verantwortlich \\
\hline $\begin{array}{l}\text { Plan und chirur- } \\
\text { gisches Vorgehen }\end{array}$ & Chirurg \\
\hline $\begin{array}{l}\text { Kommunikation } \\
\text { innerhalb des Teams }\end{array}$ & $\begin{array}{l}\text { Chirurg und } \\
\text { restliches Team }\end{array}$ \\
\hline $\begin{array}{l}\text { Checkliste der } \\
\text { Ausrüstung }\end{array}$ & $\begin{array}{l}\text { Instrumen- } \\
\text { tierende/r } \\
\text { Chirurg }\end{array}$ \\
\hline $\begin{array}{l}\text { Time-out-Check } \\
\text { vor Schnitt }\end{array}$ & $\begin{array}{l}\text { Chirurg und } \\
\text { restliches Team }\end{array}$ \\
\hline
\end{tabular}

\section{Chirurgisches Vorgehen und OP-Zeichnung durch einfache „Overlay“-Technik}

Dieser Schritt ermöglicht es dem Chirurgen, durch Studium der Röntgen- und evtl. CT-Bilder jede Fraktur genau zu erfassen. Dabei sollen jedes Fragment erkannt und dessen Umrisse und Lage auf Transparentpapier gezeichnet werden. Durch Überlagern und achsengerechte Ausrichtung der einzelnen Fragmente kann die Fraktur auf dem Papier rekonstruiert werden. Als Nächstes wird eine Schablone des gewählten Implantats auf den wieder aufgebauten Knochen gelegt und die genaue Länge und Form bestimmt. Dies erlaubt die Abfolge der einzelnen weiteren Schritte, z. B. die exakte Einbringung der Implantate und Schrauben festzulegen.

\section{Dies erfolgt in 3 Schritten:}

Schritt 1: Vom Röntgenbild der gesunden Gegenseite werden die Umrisse des Knochens gezeichnet (Abb. 1). Dies ergibt ein 2-dimensionales Bild des intakten Knochens. 


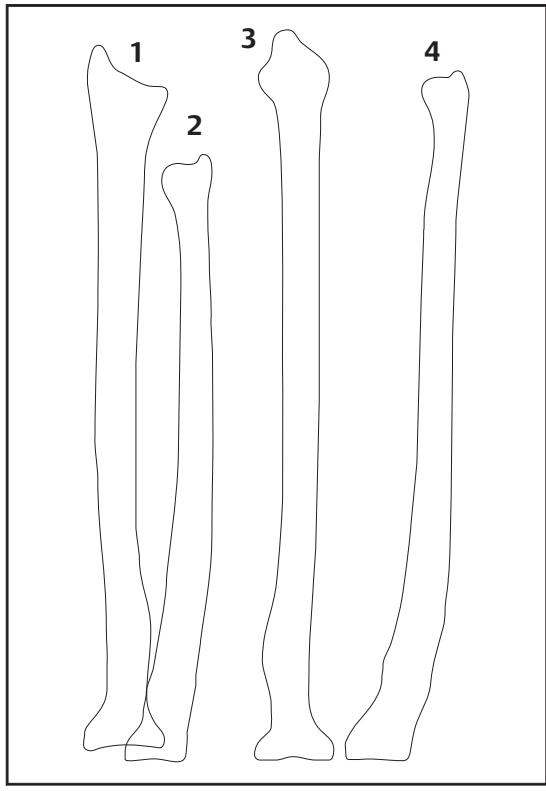

Abb. 1 Zeichnen der Konturen auf Transparentpapier vom Röntgenbild des intakten Knochens der Gegenseite. 1 = AP radius, $2=$ AP ulna, 3 = Lateral radius, 4 = Lateral ulna.

Schritt 2:. Die einzelnen Fragmente des gebrochenen Knochens werden nun auf den intakten Knochen gelegt und so „rekonstruiert" (Abb. 2a, b). Es wird mit den intakten Hauptfragmenten begonnen und die restlichen dann hinzugefügt.

Schritt 3: Der auf Transparentpapier rekonstruierte Knochen wird nun auf die Schablone des Implantats gelegt, die dieselbe Vergrößerung wie der Knochen haben muss (Abb. 3a, b).

Mit einer anderen Farbe kann nun die Lage des Implantats, z.B. einer Platte, aufgezeichnet werden. Danach wird die Reihenfolge und Richtung der einzelnen Schrauben und deren Funktion markiert (Abb. 4).

Schließlich beschreibt der Chirurg seinen Plan und Vorgehen. Dies erlaubt dem Team, den Umfang und die einzelnen Schritte des Eingriffs zu verstehen und sich entsprechend vorzubereiten. Der präoperative Plan muss dabei die gewünschte Lagerung des Patienten sowie den speziellen Operationstisch beinhalten, aber auch, ob eine Blutsperre erwünscht ist und ob und an welcher Stelle allenfalls eine autologe Knochenentnahme geplant ist.

Auch sollte der Ort bzw. die Lage des Hautschnitts festgelegt und die geplanten Repositionsmanöver und Instrumente z.B. mittels Distraktors erwähnt werden. Für den Wundverschluss benötigt

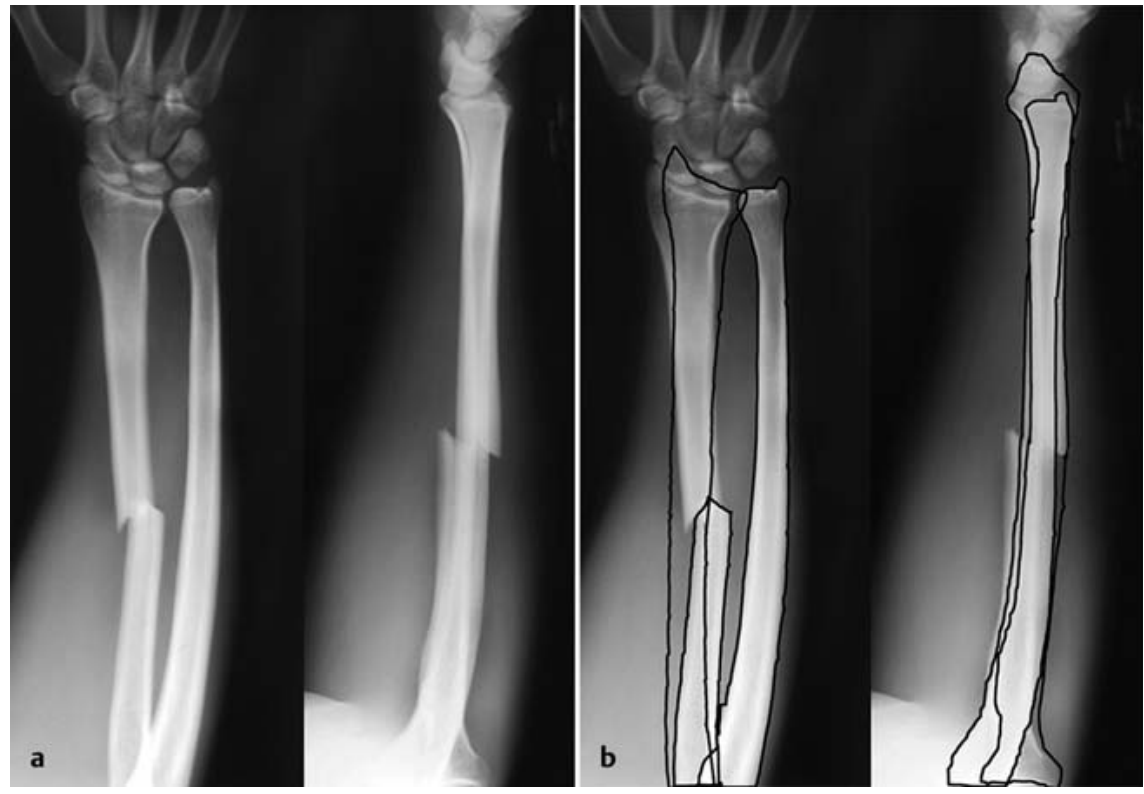

Abb. 2 a und b a Röntgenbild (a.-p. und lateral) des gebrochenen Vorderarms. b Die Zeichnung des ungebrochenen Knochens wird auf die Bilder der frakturierten Seite gelegt. Die Fragmente werden auf dem Transparentpapier „reponiert“.

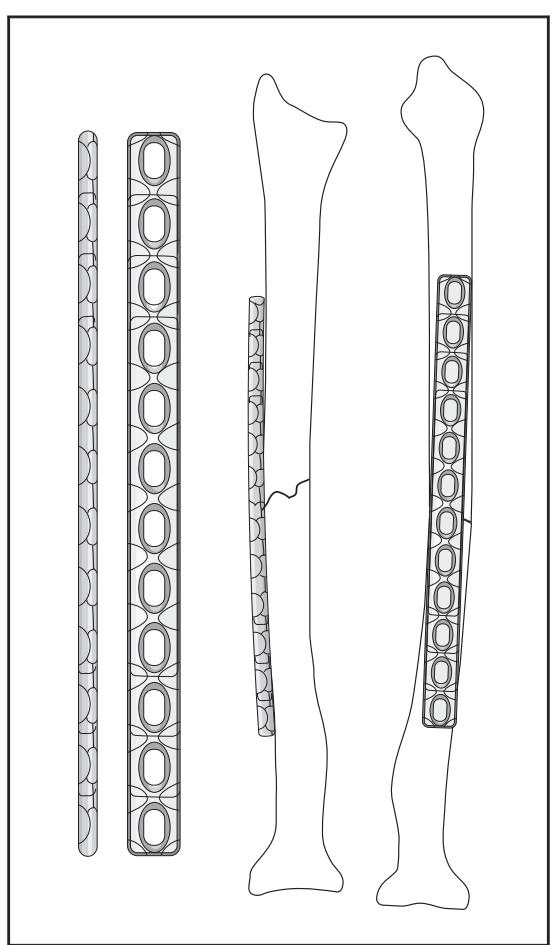

Abb. $\mathbf{3}$ a und $\mathbf{b}$ a Die Schablone einer 3.5-LCDCP wird nun ausgewählt. b Die Umrisse der Platte werden nun auf den frakturierten Knochen übertragen.

das Team Informationen, wie z. B. welche Drainagen, Nahtmaterialien, speziellen Verbände etc. benötigt werden. Der geschriebene OP-Plan wird am besten neben den Röntgenbildern für alle einsehbar aufgehängt.

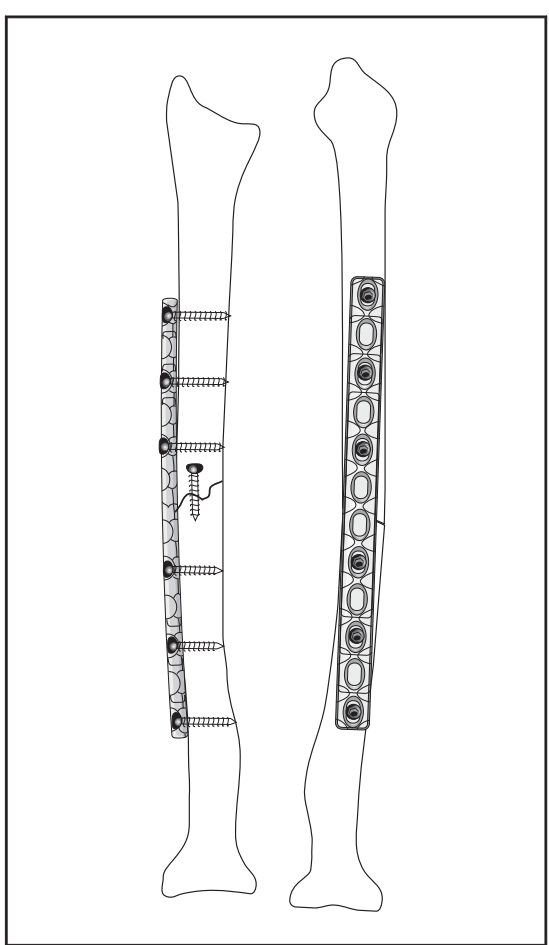

Abb. 4 Einzeichnen der verschiedenen Schrauben und Festlegen des Operationsplans in einzelnen Schritten.

\section{Digitale präoperative Planung}

Nachdem vielerorts die Röntgenaufnahmen nur noch in digitaler Form auf dem Bildschirm verfügbar sind, gibt es auch immer mehr Software, die eine ähnliche Planung am Bildschirm erlaubt. Diese 


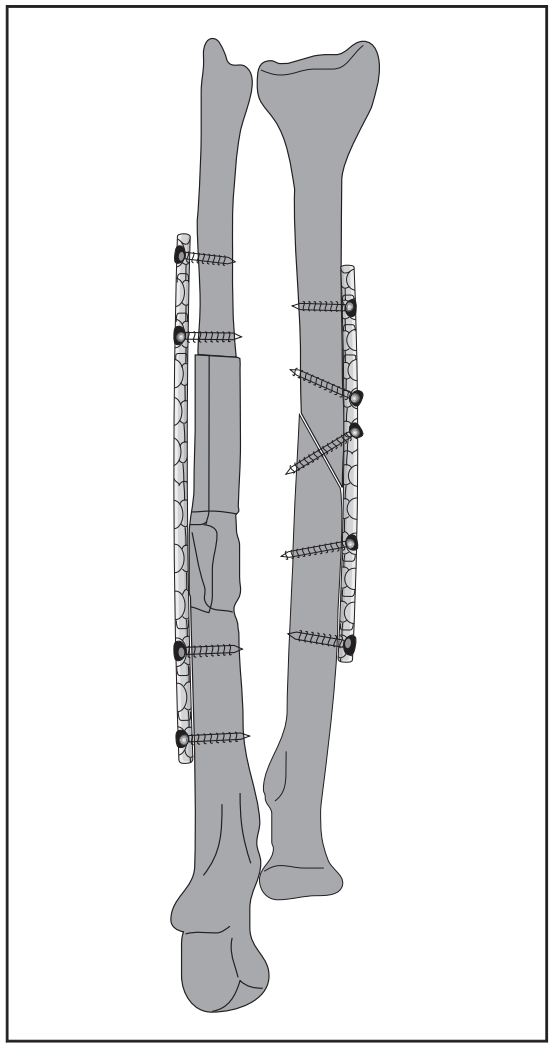

Abb. 5 Digitaler Operationsplan.

Pläne können dem Operationssaalteam elektronisch übermittelt werden (Abb. 5).

\section{Kommunikation}

Gute Kommunikation ist eines der wichtigsten Elemente bei jedem Eingriff und oft entscheidend für dessen Erfolg.

Das Erstellen eines formellen Operationsplans erlaubt dem Chirurgen, seine Taktik und Bedürfnisse dem Team konkret zu vermitteln, was schließlich auch bedingt, dass das Team seine Ansprüche mitteilt und gegebenenfalls erfahrenes Instrumentierpersonal zur Verfügung stellt. Im Weiteren werden die Bereitstellung aller erforderlichen Instrumente und eine vollständige Implantatauswahl ermöglicht sowie die Verfügbarkeit von speziellen Geräten - z.B. spezieller OPTisch oder Cell Saver - garantiert.

Kommunikation muss immer in beiden Richtungen möglich sein, sodass bei Fragen, Zweifeln oder Unklarheit das OPPersonal rückfragen kann, um sich zu vergewissern, das Richtige zu tun. Wenn sich alle Teammitglieder bemühen, proaktiv vorauszusehen und sich an der Planung beteiligen, können viele Probleme zeitgerecht gelöst und leicht übersehbare Details erkannt werden, was den

Tab. 1 Dr. Schelkuns präoperativer Plan und Checkliste, die er dem Team vorlegt.

\begin{tabular}{|c|c|c|}
\hline \multicolumn{3}{|l|}{ Date of surgery: } \\
\hline \multicolumn{3}{|l|}{ Procedure: } \\
\hline $\begin{array}{l}\text { Position of patient: } \\
\text { - Supine } \\
\text { - Prone } \\
\text { - Lateral } \\
\text { - Beach chair }\end{array}$ & $\begin{array}{l}\text { - Hip grip positioner } \\
\text { - Bean bag } \\
\text { - Axillary roll } \\
\text { - Foam headrest }\end{array}$ & $\begin{array}{l}\text { - Bump under hip } \\
\text { - Roll under shoulder }\end{array}$ \\
\hline $\begin{array}{l}\text { Table: } \\
\text { - Regular } \\
\text { - Fracture table } \\
\text { - Hand table }\end{array}$ & $\begin{array}{l}\text { - Radiolucent extension } \\
\text { - Spine table } \\
\text { - Sterile finger traps and } \\
\text { weights }\end{array}$ & $\begin{array}{l}\text { - Blue foam femoral pillow } \\
\text { - Blue foam tibia cushion } \\
\text { - Foam elbow cushion }\end{array}$ \\
\hline $\begin{array}{l}\text { X-ray: } \\
\text { - Image intensifier }\end{array}$ & - C-arm & - Portable \\
\hline $\begin{array}{l}\text { Drape pack: } \\
\text { - Extremity pack } \\
\text { - Extremity drape } \\
\text { - Bilateral extremity drape }\end{array}$ & $\begin{array}{l}\text { - Shoulder pack } \\
\text { - Hip pack } \\
\text { - Isolation drape }\end{array}$ & $\begin{array}{l}\text { - Adhesive drape } \\
\text { - Tourniquet } \\
\text { - Sterile tourniquet }\end{array}$ \\
\hline $\begin{array}{l}\text { Primary instrument set: } \\
\text { - Hand and foot } \\
\text { - Arthroscopy set } \\
\text { - Major bone set }\end{array}$ & $\begin{array}{l}\text { - Plastics set } \\
\text { - Minor bone set } \\
\text { - Hip set }\end{array}$ & $\begin{array}{l}\text { - Micro instruments } \\
\text { - Bone graft set-up on stand }\end{array}$ \\
\hline
\end{tabular}

Power tools:

- Small battery power drive set

- Large battery power drive set

- Radiolucent right angle drive for small battery drill

- Oscillating attachment for small battery drill

- High-speed burr

- Oscillating saw

- Micro-air saw

Special equipment:

- K-wires

- Osteotomes, standard - Microscope

- Skin hooks

- Probe

- Small knife handle

- Osteotomes, small

- Lead hand

- Blades: \#

- Heavy pin (bolt) cutters

- Small-hand retractors

- Hohmann retractors

- Currettes, large

- Currettes, small

$\begin{array}{ll}\text { Dressing: } & \\ -4 \times 4 \text { gauze } & - \text { Bias cut stockinette } \\ -2 \times 2 \text { gauze } & - \text { All cotton elastic wrap } \\ - \text { Fluffs } & - \text { Foam tape } \\ \text { - Soft roll } & - \text { lodine ointment } \\ \text { - ABD } & - \text { Cast cart } \\ & - \text { Cradle sling }\end{array}$

Individual instruments:

- Soft tissue washers

- Wire mounts

- Calcaneal plates

Other:

Fig 2.8-6 Example of a preoperative planning instrument and equipment list used to communicate with team members

ganzen Ablauf des Eingriffs harmonisch und zugleich sicherer für den Patienten gestaltet.

\section{Checkliste der Ausrüstung}

Vielerorts gibt es eine Datei, in der für jeden Chirurgen seine Präferenzen registriert sind, was für Routineeingriffe durchaus brauchbar ist, trotzdem sollte 
Tab. 2 Beispiel einer Time-out-„, checkoff"-Liste.

\begin{tabular}{l} 
Preoperative planning step \\
\hline Responsible member \\
\hline Patient name? \\
\hline Surgical side? \\
\hline Procedure? \\
\hline Informed consent signed by surgeon? \\
\hline Operative consent signed by patient/ \\
guardian? \\
\hline Allergies? \\
\hline Antibiotics given? \\
\hline Pressure points padded? \\
\hline Axillary roll (if lateral position) \\
\hline Safety strap? \\
\hline $\begin{array}{l}\text { X-rays/MRI up?-name correct and most } \\
\text { recent ones? }\end{array}$ \\
\hline Instrument list checked? \\
\hline Procedure briefing with team \\
\hline Questions/concerns by anyone in the \\
room? \\
\hline Music \\
\hline Inject portals \\
\hline Scrub time \\
\hline
\end{tabular}

Fig 2.8-7 Example of a time-out check-off list

bei speziellen Operationen immer ein separater Plan aufgestellt bzw. angefordert werden. Es ist beruhigend und spart viel Mühe, Ärger und Zeit, wenn alles notwendige Material bei OP-Beginn bereitsteht. Im Gegenzug kann mangelnde
Planung und Ausrüstung für alle Beteiligten frustrierend und demotivierend sein. Die Liste der Ausrüstung des Autors ist in Tab. 1 aufgeführt.

\section{Präoperativer „Time-out“-Check vor Schnitt}

Es ist absolut unerlässlich, dass vor dem Hautschnitt nochmals überprüft wird, ob der geplante Eingriff beim richtigen Patienten, an richtiger Stelle und auf korrekter Seite durchgeführt wird. Fehler passieren immer dann, wenn Abkürzungen genommen werden. Viele Spitäler fordern heute offiziell solche „Timeout"-Checks, um den Patienten Sicherheit zu garantieren. Solche Checks beanspruchen wenig Zeit und müssen schriftlich festgehalten werden.

Eine Möglichkeit, diese Prozessabläufe zu vereinfachen, ist die Einführung einer „Check-off“-Liste, ähnlich wie dies in der Aviatik Routine ist. Der Autor - ein Privatpilot - braucht regelmäßig eine solche Liste (Tab. 2), die im Operationssaal aufgehängt wird. Nachdem der Patient fertig gelagert und vorbereitet ist und jedermann seine Aufgaben erledigt hat, verlangt der Chirurg das „Time-out“. Es wird eine Person bestimmt, die zunächst Name etc. des Patienten kontrolliert. Danach wird vom Chirurgen jeder Punkt auf der Liste aufgerufen und verbal durch die verantwortliche Person bestätigt. Dies dauert ungefähr eine halbe Minute und erhöht nicht nur die Patientensicherheit, sondern verbessert auch die Kommunikation im Team. Dies ist der Moment für Fragen und erlaubt jedem Teammitglied, berechtigte Zweifel noch vor OP-Beginn anzubringen. Erst nach diesem letzten Check wird der Chirurg sich zur chirurgischen Händedesinfektion begeben.

\section{Schlussfolgerungen}

Eine erfolgreiche operative Frakturbehandlung beinhaltet eine Reihe von komplexen Abläufen und Prozessen, die viele Personen einer chirurgischen Klinik beanspruchen. Eine sorgfältige Vorbereitung und Orientierung über jeden Schritt eines Eingriffs sind dabei besonders wichtig für eine reibungslose und erfolgreiche Durchführung der Operation. Unterlässt der Chirurg diese Vorbereitung, so riskiert er, den Eingriff unnötig zu verlängern und den Erfolg zu kompromittieren. Es entstehen Ärger und Verzögerung, wenn das gewünschte Implantat oder Instrument nicht vorliegt. Andererseits wird bei vorliegendem Plan, aber mangelnder Kommunikation innerhalb des Teams sehr leicht Frustration aufkommen, was wiederum ein Risiko für die Patientensicherheit und den Operationserfolg darstellt.

\section{Literatur}

${ }^{1}$ Rüedi TP, Buckley RE, Moran CG. AO Principles of Fracture Management. 2nd ed. Stuttgart, New York: Thieme; 2007

2 Mast J, Jacob R, Ganz R. Planning and Reduction Technique in Fracture Surgery. Berlin, Heidelberg, New York: Springer; 1989

\section{Steven Schelkun, MD}

800 Pomona Avenue

Coronado, CA 92118-2340

USA 